\title{
Mathematics in the spirit of Joe Keller
}

\author{
J. R. OCKENDON ${ }^{1}$ and B. D. SLEEMAN 2,3 \\ ${ }^{1}$ Mathematical Institute, University of Oxford, Oxford, OX2 6GG, UK \\ ${ }^{2}$ School of Mathematics, University of Leeds, Leeds, LS2 9JT, UK \\ ${ }^{3}$ Division of Mathematics, University of Dundee, Dundee, DD1 4HN, UK
}

Over the two days 2-3 March 2017, about 80 mathematicians and friends gathered in Cambridge to celebrate the life and work of Joseph Bishop Keller (1923-2016), one of the pre-eminent applied mathematicians of the 20th century. Joe, as he was known throughout the world, made pioneering contributions to a wide range of natural phenomena and developed fundamental mathematical techniques with which to understand them. Twentyfour talks were presented at the meeting, given by mathematicians who have either worked with Joe or have been influenced by his work ${ }^{1}$. Rather than summarise each presentation, we have collated all the contributions under the headings of waves, fluids, solids, chemistry and biology, and finally some history.

A more personal account of the meeting by Hilary Ockendon can be found in Mathematics Today, Vol 53, No. 4, Aug. 2017.

Before we start, here is Joe's idea of a good applied mathematics problem:

A problem should have a quantitative aspect, be neither too easy nor too hard, the underlying mechanism should be understood and the solution, if found, should be enlightening and important.

\section{Waves}

Among all of Joe's contributions to applied mathematics perhaps his crowning achievement is his theory of geometrical diffraction (GTD). GTD goes beyond the theory of geometrical optics and provides a systematic understanding of how rays are diffracted by edges, corners, the vertices of boundary surfaces, or graze such surfaces, and also how rays penetrate shadow regions. The theory is widely used to describe high-frequency wave propagation in acoustics, electromagnetic theory, and even quantum mechanics. In 2001 and 2004, Joe and colleagues made an early excursion into the numerical implementation of GTD using finite and boundary element methods. Recently, Hewett and colleagues have developed hybrid numerical asymptotic methods which combine finite and boundary element methods with rigorous two-dimensional high-frequency asymptotics based on GTD to solve transmission problems and acoustic obstacle scattering problems

1 Names highlighted in boldface refer to those who gave presentations and the reader is invited to locate them both as PDF's and Video from the web site https:www.turinggateway.cam.ac.uk/event/tgmw44/programme. 


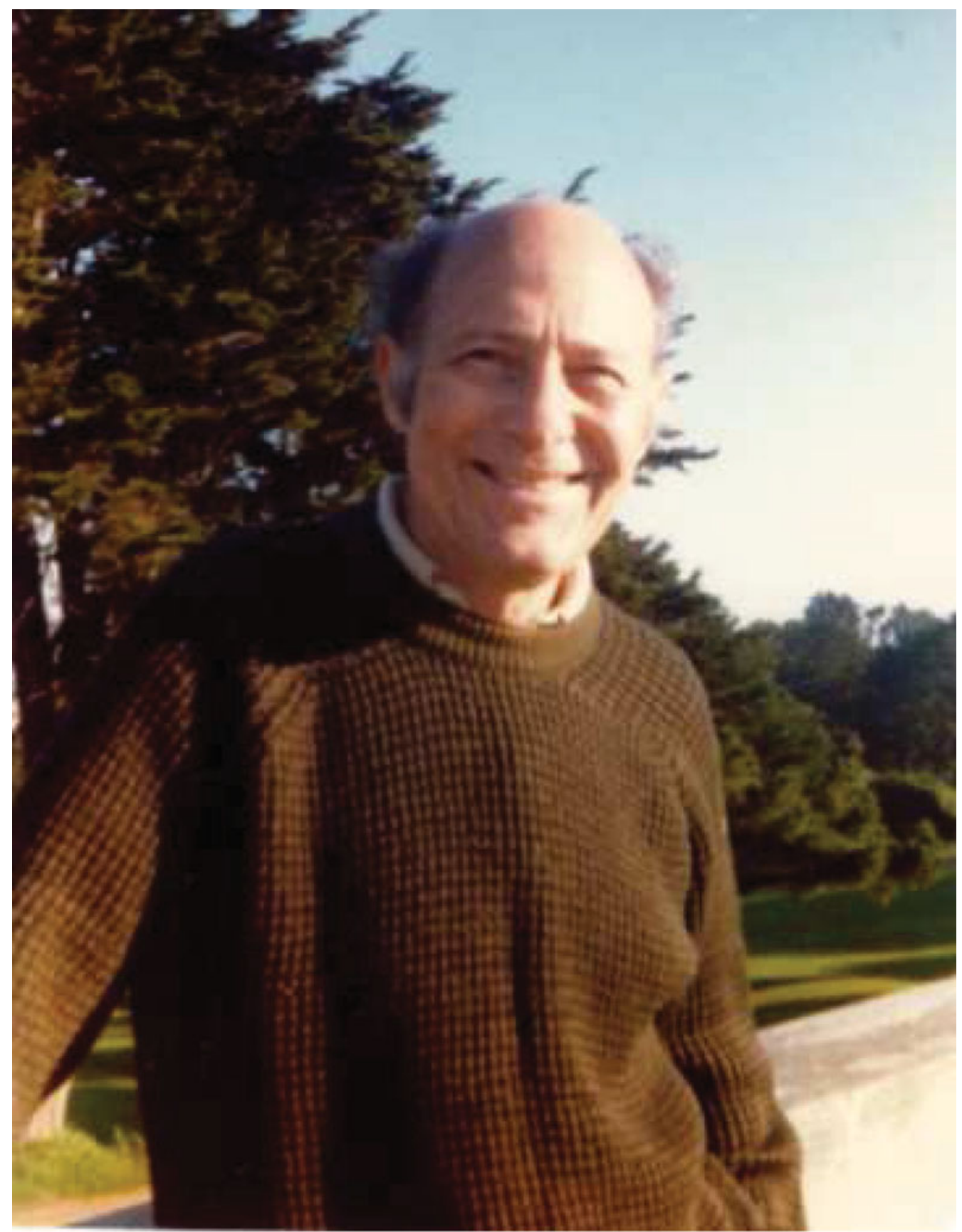

Joseph B. Keller. 


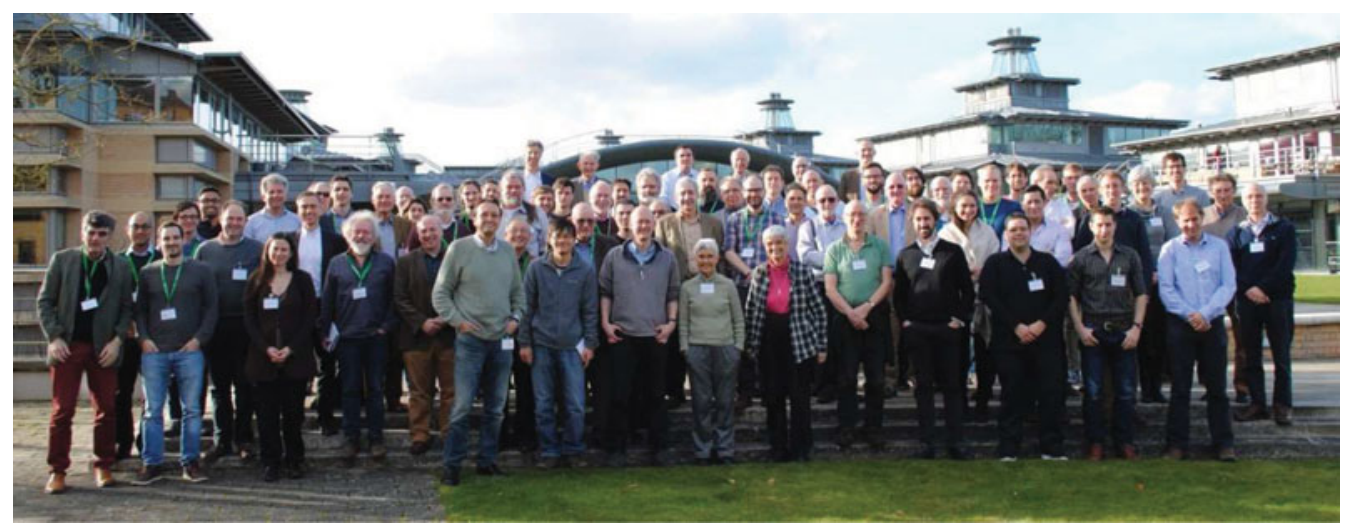

Conference participants.

involving both convex and non-convex polygons. There are numerous open problems including the extension of hybrid numerical asymptotic methods to three dimensional and the development of GTD in relation to multiple scattering.

One of the main difficulties in the numerical solution of obstacle scattering problems is that of computing radiating waves in unbounded domains. Joe showed how to overcome the difficulty by the introduction of "exact non-reflecting" boundary conditions on a finite surface, usually a ball, surrounding the scatterer. This method is numerically intensive and Grote has developed new boundary conditions that are accurate and numerically tractable.

Despite the power of GTD, a wide number of so-called "canonical problems" seem to defy exact analytic solution. One such problem, revisited by Rawlins is that of determining the radiated far field produced by an electrically polarised line source situated at the vertex of a right-angled dielectric wedge. He shows that a good first-order approximation to the scattered electric field, can be obtained by use of a double complex Laplace transform of the integral representation of this field and then employing a Neumann series expansion. This method avoids the traditional use of the double Wiener-Hopf technique.

The investigation of mirages, such as those seen of distant objects reflected in a road on a hot day, is one of those problems which fall into the category of Joe's philosophy of a good problem. However, it is one which seems to have escaped Joe's enquiring mind. In 1959 Raman and Pancharatnam argued that the phenomenon can be correctly modelled on the basis of the theory of geometric optics. A year later Raman reported disquiet about the use of geometrical optics as an explanation regarding it as "inadequate and unsatisfactory". Berry returns to the problem and shows that a simple limiting process in which the smoothly varying refractive index is approximated by a stack of discrete layers resolves any misconceptions and reveals the underlying importance of the Airy function.

\section{Fluids}

Although Joe's theories of diffraction have most frequently been applied to stationary media, they have had much impact in the field of aero-acoustics, especially to flows past 
thin wings and blades and on ducts of finite length. When plane acoustic waves are incident on such obstacles, the scattered field is dominated by a combination of what are now known as "Keller cones" of diffracted rays. As reported by C. J. Chapman, this representation, which can, for example, lead to hyperbolic umbilics, has great value in understanding otherwise confusing experimental measurements.

Joe's work on fluids covered statics as well as dynamics and led to a generalisation of Archimedes Principle to include the effects of surface tension (the net force resulting from surface tension is the weight of fluid displaced by the meniscus). But does this help to explain why pond skaters have hairy legs? As shown by Vella, even the analysis of the meniscus on a thin cylinder moving normally in and out of water leads, using Joe's work on the rupture of inviscid jets, to new scaling laws depending on the geometry of the cylinder.

Dynamic theories of immersion are more complicated, even for the problem of hydrophobic particles or drops bouncing on a horizontal water surface. An interesting new systematic model for this phenomenon was proposed by Milewski and Galeano-Rios, together with an ingenious numerical solution. It has been suggested that, since the particle motion depends on the waves it generates, this configuration might be helpful in understanding wave/particle duality in quantum mechanics. Joe was very interested in macroscopic analogues of the equations of quantum mechanics and always ready to chat about the possibilities.

Inviscid free surface flow under gravity has been the subject of a long-standing collaboration between Joe and Vanden-Broeck covering two-dimensional Stokes waves, nozzle flows, and bubble rise. Particular attention has been paid to limiting cases in which corners appear in the free boundary, either at separation from a fixed boundary or in the absence of fixed boundaries. As often happens in theoretical mechanics, three-dimensional axisymmetric generalisations can produce oscillatory variations that are absent in two dimensions. This has recently been shown to happen in several of the previous studies, leading to unexpected profiles in different Froude number regimes and, in particular, in the search for annular Stokes waves with a $120^{\circ}$-corner.

The motion of bubbles in the presence of surfactants is central in chemical engineering and in 2004, Joe proposed some interesting boundary conditions to be imposed on models for such bubbles. Papageorgiou showed how the computation of even a moderately comprehensive model for the surface kinetics results in a complicated pattern of parameter regimes, with no clear implications for the bubble surface rigidity which controls its mobility and the resulting mass transfer. Hopefully, the use of simple boundary conditions will lead to better understanding at least in the case of slow flow.

The flow of plasma in the solar corona or a tokomak is an extreme example of fluid dynamics and new phenomena can occur, even for unidirectional flow in a channel. Dellar considered such flows when the applied magnetic field is strong enough to make the stress tensor in the fluid anisotropic. This makes the resulting asymptotic analysis more intricate than for traditional Hartmann flows and, as often happens near plasma boundaries, an awkward initial/boundary value problem in the boundary layers. Fortunately, conversations on the porch of Walsh Cottage at Woods Hole Oceanographic Institution elucidated the relevant asymptotic structure. 


\section{Solids}

In the 1960's, Joe's effortless modelling and analysis of "thin" solids put him in a perfect position to shed new light on bifurcation theory, which was all the rage, especially at the Courant Institute of Mathematical Sciences.

While buckling of unconfined straight rods is easy to study, the buckling of, say, confined rods or rings is much more interesting. A version of the latter was addressed by Mullin who had devised the ingenious experiment of putting a buoyant elastic ring on the surface of a conical container of liquid and slowly releasing the liquid from the bottom of the cone. Whereas Joe had discovered a beautiful series of pitchfork bifurcations as the pressure on an unconfined ring is increased, the constraint provided by the walls of the cone forces the response diagram to comprise only disconnected branches of solutions.

Motivated by plant mechanics, the configuration of a hanging heavy clamped rod was discussed by Goriely. While phase plane analysis makes it easy to classify the possible equilibrium shapes, and analysis of the second variation of the energy is not so easy. However, the stability of any particular configuration can often be decided by a simple inspection of the geometry of the phase plane.

Multiple interacting rods, such as hair, lead to more difficult modelling challenges. Goldstein used ideas from statistical physics to construct an energy functional for hair that leads to an equivalent single fibre model for, say, a ponytail. Naturally, this model is more complex than that used to such effect by Joe in his famous ponytail paper, and it paves the way for a more systematic analysis of the inter-fibre forces that act on assemblies of, maybe, $10^{5}$ hairs. These forces are different in the study of another of Joe's prototypes, the modelling of ropes and shoelaces. As described by Maddocks, the relevant analysis of the frictional forces, which generally act along two contact lines for any particular fibre, leads to a complicated system of functional delay equations.

Yet, another fundamental research area that Joe opened up in the 1960's was that of the homogenisation of the material properties of composites when measurements are made on a much greater scale than the length scale of the inhomogeneity. As described by Craster, Joe derived a fiendishly clever exact solution for the simplest electrostatic checkerboard problem with different constant conductivities in the black and white squares. The homogenised conductivity is the geometric mean of these two conductivities and this result has proved to be a benchmark for many subsequent homogenisation studies. Moreover, with Joe's encouragement, an exact solution has since emerged for doubly periodic tilings.

A quite different homogenisation problem that was solved, using an extension of Joe's strategy for composites with strongly contrasting material properties, is that of the Faraday cage whose main application is, of course, in electromagnetism. As explained by S. J. Chapman, a systematic matched asymptotic expansion analysis reveals that the homogenised cage boundary supports a jump in the field that is proportional to the difference between the potential of the field and the unknown potential of the wires. For effective screening by a circular cage, the ratio of the wire radius to the cage radius should greatly exceed $e^{-n} / n$, where $n$ is the number of wires.

A final mechanics problem that would have surely attracted Joe's attention was described by Howison. This concerns the Estonian recreation of kiiking, meaning standing or 
squatting on the seat of a rigid swing in such a way as to make repeated $360^{\circ}$ rotations. A Keller approach of trying bang-bang control of the squatting/standing at the top/bottom of each cycle leads to a strategy for minimising the energy necessary to kiik. Multiple scales analysis is then needed to see what happens after, say, 50 cycles. All this insight comes without any reliance on heavy theories of optimal control, which epitomises the study of theoretical mechanics in Joe's style.

\section{Chemistry and biology}

Liesegang rings are observed when a drop of silver nitrate solution is placed on a surface of potassium dichromate gel. The resultant precipitate of silver dichromate forms concentric patterns of rings. Such rings are observed in sedimentary rock deposits. Joe and Sol Rubinow developed a theory of Liesegang rings based on a system of reaction diffusion equations and demonstrated that precipitation rings occur when the product, arising from the chemical reaction reaches a certain supersaturation value. Fowler revisits the phenomenon and clarifies the Keller-Rubinow theory by the introduction of a switching mechanism which helps to explain the formation of precipitation bands.

Although Joe did not directly work on tumour angiogenesis, he was certainly aware of the need to understand the growth of tumours through his work on skin tumours in mice (see Whittemore). Angiogenesis is the mechanism by which isolated avascular tumours may develop through the initiation and recruitment of a neovascular network of capillaries. This in turn provides the tumour with its own blood supply which results in explosive tumour growth and subsequent metastases to distant regions of the body. Many continuum models have been developed to describe the mechanisms underlying angiogenesis. Bonilla generalises this approach by modelling tumour angiogensis in terms of stochastic partial differential equations and encounters a connection with soliton-like behaviour during capillary vessel growth.

The study of lenses has a long history with fundamentals set down by Hamilton, Maxwell, and Keller. Rubinstein addresses the practical problems of spectacle lens design from the optician's viewpoint. Spectacle lens design needs to take into account (i) the eye is not radially symmetric, (ii) the eye scans the visual field and so the lens must accomodate vision from many directions, and (iii) the pupil is relatively small and so only a small wavefront, generated by each source needs to be considered for any gaze direction. Rubinstein illustrates the importance of Hamilton's eikonal function and its computation by Maxwell and latterly by Joe and Herb Keller and how their ideas are now central to optical lens design.

An area of physiology in which Joe, together with John Rinzel, made a pioneering contribution was in modelling nerve impulse transmissions (see Sleeman) in unmyelinated nerve fibres. A basic model is the so-called FitzHugh-Nagumo system of coupled nonlinear partial differential equations. Joe recognised a crucial nonlinear term appearing in the system which, while having desirable qualitative properties, made the analysis of nerve wave pulses intractable. In typical Keller fashion, Joe replaced this term by a piecewise linear one which led to a comprehensive phase space analysis using matched asymptotic expansions. This device elucidated the important characteristics of the nerve impulse including its stability and threshold behaviour. 


\section{The Keller spirit}

The Courant Institute in the 1960's and 70's was internationally pre-eminent with a faculty comprised of some of the most outstanding mathematicians of the 20th century. Richard Courant was the Scientific Adviser to the Governing Council and Joe was the Director of the Division of Electromagnetic Research. Louis Nirenberg was Director and Head of the All-University Departments of Mathematics, Peter Lax headed Computing and Applied Mathematics, Fritz John directed Mathematics and Mechanics while Harold Grad was Director of the Magneto-Fluid Dynamics Division. Other faculty members included Alexander Chorin, Kurt Friedrichs, Paul Garabedian, Morris Kline, Wilhem Magnus, Jürgen Moser, James Stoker and Heinz-Otto Kreiss. There were seminars conducted everyday presented in a very informal manner and the Faculty members were usually accessible for discussions whether in offices or in the Lounge on the top floor of the Courant Institute. Sleeman was a visiting member of Joe's group and was greatly influenced by Joe's enthusiasm and ideas which led to his investigations into the rigorous justification of the geometrical theory of diffraction, an interest in nerve impulse transmissions as well as inverse problems.

While Joe was inventing the geometrical theory of diffraction, work was being carried out in the Soviet Union and Smyshlyaev provided fascinating insights into the research being carried out in Leningrad during this period. There was no mathematical "Iron Curtain", for while the New York Times or the Washington Post were not available, Communications in Pure and Applied Mathematics certainly was. V. A. Fock was a founder of the Soviet School on diffraction, and his "principle of locality" highlighted the importance of appropriate canonical problems, as does GTD. With Leontovich, this led to the "method of parabolic equations" used to describe grazing rays and in which combinations of Airy functions play a central role in matching geometrical optics and obtaining decay estimates as one proceeds into the shadow of the scattering obstacle. To cite V. M. Babich, another leader of the St Petersburg school, "The Influence of Joseph B. Keller's ideas on the Soviet/Russian diffraction school was and remains very significant".

The meeting was delighted and honoured that Alice Whittemore, Joe's widow, gave the meeting insight into the qualities of a great human being. Joe was a man of great creativity and curiosity, imbued with discernment, wit, and "joie de vivre". Although Joe received many distinguished honours worldwide, he led a simple and informal life. Indeed there was concern about what Joe should wear when he went to Washington to receive the U.S National Medal of Science from President Ronald Reagan. He hurriedly went out at Woods Hole and bought a suit. From that time on, whenever Joe was to attend a formal occasion, it had to be decided whether the occasion necessitated the "The Reagan Suit" or not. Joe was also an outdoor man and he and Alice enjoyed many adventures of hiking, skiing, exploring caves, and good food. Many applied mathematicians around the world are familiar with Joe's wit, particularly in regard to inverse problems, in which the answer to a question is known but the question is not known. Such problems are vitally important in areas ranging from medical imaging to oil recovery and as described by Fokas, the unknown shape that creates an image can often be 


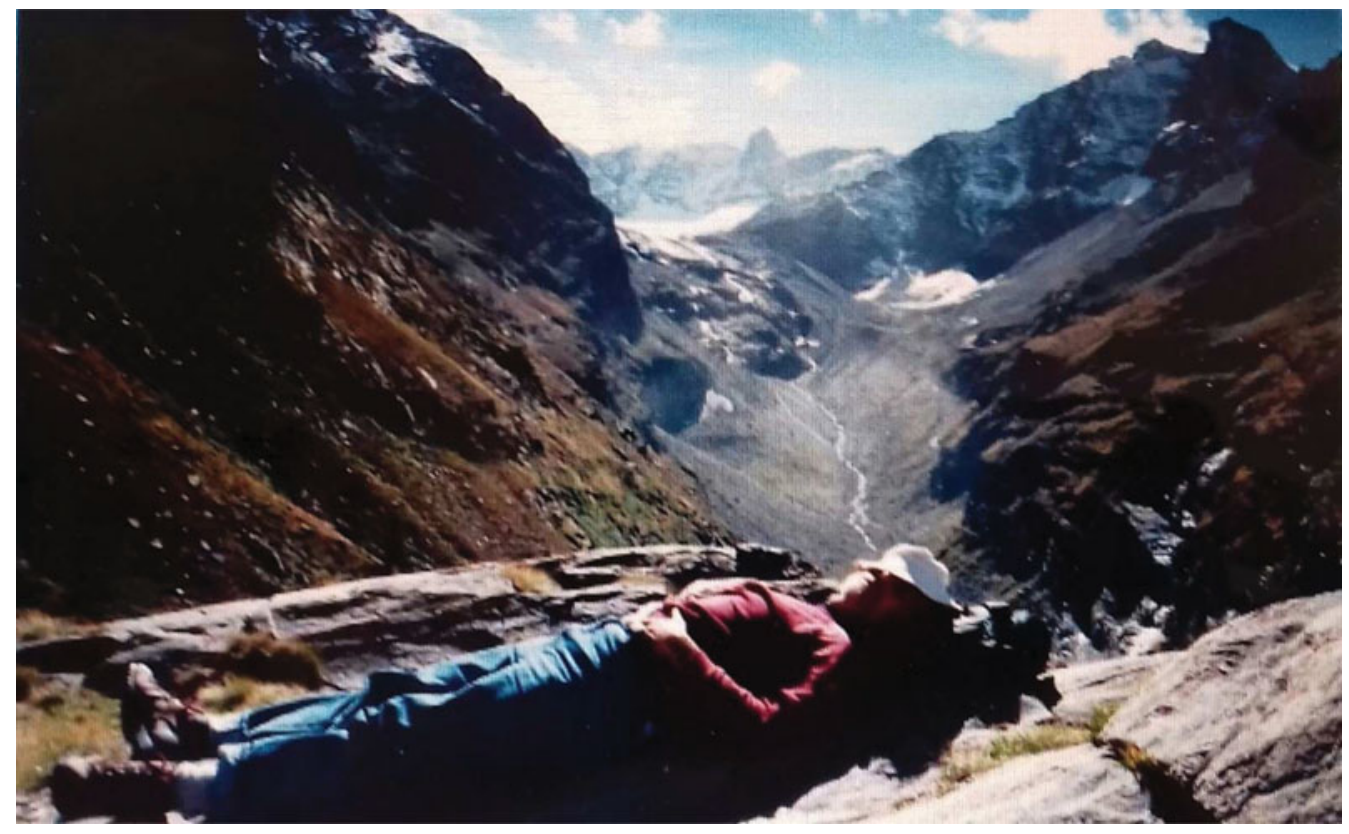

Problem solved, Joe relaxing.

reconstructed by using the Radon Transform. We conclude with one of Joe's favourite inverse problems:

To which question is the answer "Dr Livingston I presume"? 